\title{
The effect of surface treatment and
}

thermocycling on the shear bond strength

of orthodontic brackets to the Y-TZP

zirconia ceramics: A systematic review

\author{
Tamzid AHMED' \\ (i) https://orcid.org/0000-0003-2415-2204 \\ Nashid FAREEN ${ }^{2}$ \\ (iD https://orcid.org/0000-0003-4432-0746 \\ Mohammad Khursheed ALAM ${ }^{3}$ 叫 \\ (iD https://orcid.org/0000-0001-7131-1752
}

Submitted: January 11, 2021 - Revised and accepted: May 17, 2021

凶dralam@gmail.com

How to cite: Ahmed T, Fareen N, Alam MK. The effect of surface treatment and thermocycling on the shear bond strength of orthodontic brackets to the Y-TZP zirconia ceramics: A systematic review. Dental Press J Orthod. 2021;26(5):e212118.

(1) Bangladesh Dental College, Department of Science of Dental Materials (Dhaka, Bangladesh).

(2) Bangladesh Dental College, Department of Conservative Dentistry and Endodontics (Dhaka, Bangladesh).

(3) Jouf University, College of Dentistry, Department of Orthodontics (Sakaka, Kingdom of Saudi Arabia). 


\section{ABSTRACT}

Introduction: Various surface pre-treatment methods have been adapted to optimize the bonding between the zirconia ceramics and the orthodontic brackets.

Objective: This review is aimed at systematically analyzing the relevant data available in the literature, to find out the most effective and durable bonding protocol.

Methods: Database search was conducted in PubMed, Scopus, and ScienceDirect, during September 2020. The review was conducted according to the PRISMA guidelines.

Results: Based on the inclusion criteria, 19 articles were selected for qualitative analysis. Meta-analysis could not be performed due to the heterogeneity of the methodology among the studies. Most of the studies scored medium risk of bias. Compared to the untreated surface, surface pretreatments like sandblasting and lasers were advantageous. Primers and universal adhesive were mostly used as an adjunct to the mechanical pretreatment of the zirconia surface. In most studies, thermocycling seemed to lower the shear bond strength (SBS) of the orthodontic brackets.

Conclusion: Based on this qualitative review, surface pretreatments with lasers and sandblasting can be suggested to optimize the bracket bond strength. To clarify this finding, meta-analysis is anticipated. Hence, high heterogeneity of the included studies demands standardization of the methodology.

Keywords: Y-TZP zirconia. Orthodontic brackets. Shear bond strength. Surface treatment. 


\section{RESUMO}

Introdução: Diferentes métodos de pré-tratamento da superfície foram adaptados para otimizar a colagem entre os braquetes ortodônticos e as cerâmicas de zircônia.

Objetivo: A presente revisão teve como objetivo analisar, de forma sistemática, os dados relevantes na literatura, para buscar o protocolo de colagem mais efetivo e duradouro.

Métodos: As buscas foram feitas em setembro de 2020, nas seguintes bases de dados: PubMed, Scopus e ScienceDirect. A revisão foi feita de acordo com as diretrizes do PRISMA.

Resultados: Com base nos critérios de inclusão, 19 artigos foram selecionados para análise qualitativa. A metanálise não pôde ser feita, devido à heterogeneidade na metodologia dos estudos incluídos. A maior parte dos estudos apresentou risco de viés moderado. Comparadas às superfícies não tratadas, as superfícies com pré-tratamento usando jateamento ou laser apresentaram vantagem. Primers e adesivos universais foram mais usados como adjuntos ao pré-tratamento mecânico da superfície de zircônia. Na maioria dos estudos, a termociclagem parece ter reduzido a resistência ao cisalhamento dos braquetes ortodônticos.

Conclusões: Com base nessa revisão qualitativa, os pré-tratamentos de superfície com laser e jateamento podem ser sugeridos para otimizar a resistência ao cisalhamento dos braquetes. Para esclarecer esse achado, uma metanálise é necessária. Para isso, faz-se necessária a padronização da metodologia, para lidar com a alta heterogeneidade dos estudos incluídos.

Palavras-chave: Zircônia Y-TZP. Braquetes ortodônticos. Resistência ao cisalhamento. Tratamento de superfície. 


\section{INTRODUCTION}

With the increasing demand for esthetics and the introduction of the CAD/CAM devices, zirconia ceramics have gained much popularity in modern Dentistry. Zirconia claimed superiority in terms of mechanical properties, biocompatibility, precision and esthetics. ${ }^{1,2}$ The yttrium-stabilized tetragonal zirconia polycrystal (Y-TZP) is the form most commonly used in Dentistry. The material is known for the phase transformation from tetragonal to monoclinic due to stressful conditions, achieving fracture resistance. ${ }^{3}$ It has been used extensively to make inlays, onlays, crowns, post and core systems, and frameworks for porcelain fused to zirconia restorations. ${ }^{4}$ Hence, with the increasing use of zirconia restorative materials, it is becoming more common for orthodontists to bond orthodontic brackets to a Y-TZP surface. However, due to the chemical inertness and resistance to acid-etching, bonding composite resin to the zirconia surface can be challenging. Thus, various surface treatment methods - such as mechanical, chemical, or combined - have been introduced to improve the bond strength of zirconia ceramics.

The in-vitro shear bond strength (SBS) is the most commonly used test, due to its simplicity and resemblance to the shear force exerted during mastication. In addition, aiming at testing 
the durability of these methods, the material can be exposed to water at a certain temperature, simulating an oral environment for a determined period - a procedure known as thermocycling.

Previously, reviews were mostly based on the bond strength between the luting cement and the zirconium surface; overlooking the impact of orthodontic brackets. ${ }^{5-7}$ Therefore, this study is aimed to perform a systematic review of the in-vitro studies that reported the effect of different surface conditioning and aging on the shear bond strength of orthodontic brackets to the Y-TZP zirconia ceramics.

\section{MATERIAL AND METHODS}

\section{SEARCH STRATEGY}

The review was conducted by following the Preferred Reporting Items for Systematic Reviews and Meta-Analyses (PRISMA) guidelines. The research question of this review was: Which surface treatment is best suited to optimize the shear bond strength of orthodontic brackets to Y-TZP zirconia ceramics? The question was developed concerning the following PICO (population, intervention, comparison, and outcome): Y-TZP zirconia, surface treatment, thermocycling, no thermocycling, comparison between surface treatment methods with the resultant shear bond strength (SBS), respectively. 
The electronic databases PubMed, Scopus, and ScienceDirect were searched, using the following keywords: orthodontic brackets, shear bond strength, zirconium, ceramic surface, and thermocycling. Additionally, Google Scholar was searched with the same keywords. The references of the selected studies were also checked for any missing relevant studies. All searches were performed in September 2020. The search was confined to the original articles written in English language, published between the years 2001 to 2020. All the duplicates were resolved by the Endnote $X 7$ software (Thompson Reuters, Philadelphia, PA, USA). The search strategy is summarized in Table 1.

Table 1: Database search strategy.

\begin{tabular}{|c|c|c|c|c|}
\hline Name of Database & Last date of Search & Keywords & Studies found & Studies selected \\
\hline Pubmed Central & $12 / 09 / 2020$ & \multirow{4}{*}{$\begin{array}{l}\text { Orthodontic brackets, } \\
\text { shear bond strength, } \\
\text { zirconium, ceramic } \\
\text { surface, thermocy- } \\
\text { cling }\end{array}$} & 28 & 12 \\
\hline Scopus & $12 / 09 / 2020$ & & 08 & 08 \\
\hline ScienceDirect & $12 / 09 / 2020$ & & 15 & 2 \\
\hline Google Scholar & $15 / 09 / 2020$ & & 1680 & 86 \\
\hline
\end{tabular}

\section{STUDY SELECTION}

Two independent reviewers screened the titles and abstracts of all the studies found. The articles were selected according to the following inclusion and exclusion criteria:

\section{INCLUSION CRITERIA}

1) Studies that bonded orthodontic brackets to Y-TZP surface.

2) Studies that conditioned the surface before bonding. 
3) Studies with a well-designed shear bond strength test.

4) In-vitro experimental and observational studies.

5) Full-text original articles.

\section{EXCLUSION CRITERIA}

1) In-vivo studies and clinical trials.

2) Studies testing materials other than orthodontic brackets and Y-TZP zirconia, such as resins or composite cement, buccal tubes, enamel, dentine, zirconium fused to porcelain or lithium silicate infused with zirconium, etc.

The agreement between both reviewers was confirmed by Cohen's kappa statistics. ${ }^{8} \mathrm{~A}$ third reviewer was consulted in the event of any disagreement.

\section{DATA EXTRACTION}

Two reviewers independently reviewed the full-text articles and extracted the following data in tabulated form: study year, sample size, surface treatment method, adhesives and brackets used, storage, method of thermocycling, and the resultant shear bond strength (Table 2). Agreement between the reviewers was assessed by Cohen's Kappa statistics. The third reviewer's opinion was considered to resolve any disagreement. 
Table 2: Data extraction.

\begin{tabular}{|c|c|c|c|c|c|c|c|}
\hline Study & Sample & Surface treatment & Adhesive & Brackets & Storage & $\begin{array}{l}\text { Thermocy- } \\
\text { cling }\end{array}$ & $\begin{array}{l}\text { Bond strength } \\
(\mathrm{MPa})\end{array}$ \\
\hline $\begin{array}{l}\text { Akay } \\
\text { et al. }^{12} \\
(2020)\end{array}$ & 48 & $\begin{array}{l}\text { G1) Er:YAG laser (2W) } \\
\text { G2) Nd:YAG laser ( } 2 \mathrm{~W}) \\
\text { G3) Sandblasting }\left(\mathrm{SiO}_{2} \text { ) }\right. \\
\text { G4) } 9.6 \% \text { hydrofluoric acid }\end{array}$ & $\begin{array}{l}\text { Transbond } \\
\text { XT light } \\
\text { cured com- } \\
\text { posite }\end{array}$ & $\begin{array}{c}\text { Maxillary } \\
\text { central } \\
\text { incisor } \\
\text { metal } \\
\text { brackets }\end{array}$ & $\begin{array}{l}37^{\circ} \mathrm{C} \text { deion- } \\
\text { ized water for } \\
30 \text { days }\end{array}$ & $\begin{array}{c}2,000 \text { cycles } \\
\text { between } \\
5 \pm 2-55 \pm 2^{\circ} \mathrm{C} \\
\text { with } 30 \mathrm{~s} \\
\text { dwelling } \\
\text { time }\end{array}$ & $\begin{array}{l}\text { G1: } 5.5 \pm 0.79 \\
\text { G2: } 4.88 \pm 0.82 \\
\text { G3: } 7.42 \pm 0.92 \\
\text { G4: } 3.58 \pm 0.75\end{array}$ \\
\hline $\begin{array}{l}\text { Ju } \\
\text { et al. }{ }^{4} \\
\text { (2020) }\end{array}$ & 80 & $\begin{array}{l}\text { G1) Sandblasting } \\
\text { G2) Sandblasting + Clearfil } \\
\text { ceramic primer on zirco- } \\
\text { nia surface } \\
\text { G3) Sandblasting + Clear- } \\
\text { fil ceramic primer on } \\
\text { bracket base } \\
\text { G4) Sandblasting + Clear- } \\
\text { fil ceramic primer on } \\
\text { both zirconia surface and } \\
\text { bracket base }\end{array}$ & $\begin{array}{l}\text { Transbond } \\
\text { XT primer + } \\
\text { Transbond } \\
\text { XT light } \\
\text { cure com- } \\
\text { posite }\end{array}$ & $\begin{array}{l}\text { Monocrys- } \\
\text { talline } \\
\text { ceramic } \\
\text { brackets }\end{array}$ & $\begin{array}{c}\text { At } 37^{\circ} \mathrm{C} \text { and } \\
\text { humidity } \\
100 \% \text { for } 24 \mathrm{~h} .\end{array}$ & $\begin{array}{c}10,000 \text { cy- } \\
\text { cles between } \\
5^{\circ}-55^{\circ} \mathrm{C} \text { with } \\
30 \text { s dwelling } \\
\text { time }\end{array}$ & $\begin{array}{c}\text { Mean and stan- } \\
\text { dard deviation } \\
\text { not included }\end{array}$ \\
\hline $\begin{array}{l}\text { Ju } \\
\text { et al. } \\
\text { (2019) }\end{array}$ & 60 & $\begin{array}{l}\text { G1) Sandblasting + Clearfil } \\
\text { Ceramic primer + ortho- } \\
\text { dontic primer } \\
\text { G2) Sandblasting + univer- } \\
\text { sal adhesive } \\
\text { G3) Sandblasting + Clearfil } \\
\text { ceramic primer + univer- } \\
\text { sal adhesive }\end{array}$ & $\begin{array}{l}\text { Transbond } \\
\text { XT light } \\
\text { cured com- } \\
\text { posite }\end{array}$ & $\begin{array}{l}\text { Maxillary } \\
\text { central } \\
\text { incisor } \\
\text { ceramic } \\
\text { brackets }\end{array}$ & $\begin{array}{l}37^{\circ} \mathrm{C} \text { and rela- } \\
\text { tive humidity } \\
100 \% \text { incuba- } \\
\text { tor for } 24 \mathrm{~h} .\end{array}$ & $\begin{array}{l}10,000 \text { cy- } \\
\text { cles between } \\
5^{\circ}-55^{\circ} \mathrm{C} \text { with } \\
30 \text { s dwelling } \\
\text { time }\end{array}$ & $\begin{array}{c}\text { Before } \\
\text { thermocycling: } \\
\text { G1: } 9.78 \pm 1.94 \\
\text { G2: } 9.86 \pm 1.33 \\
\text { G3: } 9.16 \pm 0.78 \\
\text { After } \\
\text { thermocycling: } \\
\text { G1: } 8.16 \pm 1.78 \\
\text { G2: } 4.99 \pm 0.99 \\
\text { G3: } 4.31 \pm 1.02\end{array}$ \\
\hline $\begin{array}{l}\text { Mehmeti } \\
\text { et al. }{ }^{26} \\
(2019)\end{array}$ & 48 & $\begin{array}{c}\text { G1) } 37 \% \text { phosphoric acid } \\
\text { for } 120 \text { s + silane primer } \\
\text { G2) } 5 \% \text { hydrofluoric acid + } \\
\text { silane primer }\end{array}$ & $\begin{array}{l}\text { Transbond } \\
\text { XT primer + } \\
\text { Transbond } \\
\text { XT light } \\
\text { cure com- } \\
\text { posite }\end{array}$ & $\begin{array}{l}\text { i) Metal } \\
\text { brackets } \\
\text { ii) Poly- } \\
\text { crystalline } \\
\text { ceramic } \\
\text { brackets }\end{array}$ & $\begin{array}{l}\text { Not men- } \\
\text { tioned }\end{array}$ & $\begin{array}{c}5,800 \text { cycles } \\
\text { between } \\
5^{\circ}-55^{\circ} \mathrm{C} \text { with } \\
10 \text { s dwelling } \\
\text { time }\end{array}$ & $\begin{array}{c}\text { Metal brackets: } \\
\text { G1: } 10.85 \pm 5.84 \\
\text { G2: } 8.52 \pm 4.72 \\
\text { Ceramic } \\
\text { brackets: } \\
\text { G1: } 11.84 \pm 7.30 \\
\text { G2: } 8.99 \pm 5.36\end{array}$ \\
\hline $\begin{array}{l}\text { Cetik } \\
\text { et al. } .^{15} \\
\text { (2019) }\end{array}$ & 40 & $\begin{array}{l}\text { G1) Sandblasting + Silane } \\
\text { primer } \\
\text { G2) Er:YAG laser + Silane } \\
\text { primer }\end{array}$ & $\begin{array}{c}\text { Brack Fix } \\
\text { primer + } \\
\text { Brack 1 Fix } \\
\text { light cured } \\
\text { composite }\end{array}$ & $\begin{array}{l}\text { i) Mandib- } \\
\text { ular ante- } \\
\text { rior metal } \\
\text { brackets } \\
\text { ii) Man- } \\
\text { dibular } \\
\text { anterior } \\
\text { ceramic } \\
\text { brackets }\end{array}$ & Not done & Not done & $\begin{array}{c}\text { Metal brackets: } \\
\text { G1: } 23.29 \pm 5.34 \\
\text { G2: } 21.59 \pm 4.03 \\
\\
\text { Ceramic } \\
\text { brackets: } \\
\text { G1: } 20.06 \pm 4.05 \\
\text { G2: } 17.55 \pm 3.88\end{array}$ \\
\hline
\end{tabular}


Table 2 - continuation: Data extraction.

\begin{tabular}{|c|c|c|c|c|c|c|c|}
\hline Study & Sample & Surface treatment & Adhesive & Brackets & Storage & $\begin{array}{l}\text { Thermocy- } \\
\text { cling }\end{array}$ & $\begin{array}{l}\text { Bond strength } \\
\qquad(\mathrm{MPa})\end{array}$ \\
\hline $\begin{array}{l}\text { Douara } \\
\text { et al. }{ }^{27} \\
\text { (2019) }\end{array}$ & 45 & $\begin{array}{c}\mathrm{G} 1) \text { Sandblasting }\left(\mathrm{Al}_{2} \mathrm{O}_{3}\right)+ \\
\text { universal bonding resin } \\
\text { (Assure plus) } \\
\mathrm{G} 2) \text { Sandblasting }\left(\mathrm{Al}_{2} \mathrm{O}_{3}\right)+ \\
\text { Silane + Universal bonding } \\
\text { resin (Assure plus) + } \mathrm{Silane} \\
\mathrm{G} 3 \text { ) Sandblasting }\left(\mathrm{Al}_{2} \mathrm{O}_{3}\right) \\
+4 \% \text { hydrofluoric acid }+ \\
\text { Silane }\end{array}$ & $\begin{array}{c}\text { Transbond } \\
\text { XT primer+ } \\
\text { Transbond } \\
\text { XT light } \\
\text { cure com- } \\
\text { posite resin }\end{array}$ & $\begin{array}{l}\text { Monocrys- } \\
\text { talline } \\
\text { ceramic } \\
\text { brackets }\end{array}$ & $\begin{array}{l}37^{\circ} \mathrm{C} \text { distilled } \\
\text { water for } \\
24 \text { hrs. }\end{array}$ & $\begin{array}{c}500 \text { cycles } \\
\text { between } \\
5^{\circ}-55^{\circ} \mathrm{C} \text { with } \\
30 \text { s dwelling } \\
\text { time }\end{array}$ & $\begin{array}{l}G 1: 2.50 \pm 0.75 \\
G 2: 7.81 \pm 2.81 \\
G 3: 8.15 \pm 2.41\end{array}$ \\
\hline $\begin{array}{l}\text { Garcia-Sanz } \\
\text { et al. }{ }^{9} \\
(2018)\end{array}$ & 90 & $\begin{array}{l}\text { G1) No treatment } \\
\text { G2) Sandblasting } \\
\text { G3) Femtosecond Ti:Sap- } \\
\text { phire laser output pow- } \\
\text { er- } 300 \mathrm{~mW} \text {, inter-groove } \\
\text { distance } 60 \mu \mathrm{m} \\
\text { G4) Femtosecond Ti:Sap- } \\
\text { phire laser output pow- } \\
\text { er- } 200 \text { mW, inter-groove } \\
\text { distance } 100 \mu \mathrm{m} \\
\text { G5) Femtosecond Ti:Sap- } \\
\text { phire laser output power- } \\
40 \text { mW, inter-groove dis- } \\
\text { tance } 60 \mu \mathrm{m} \text {. } \\
\text { G6) Femtosecond Ti:Sap- } \\
\text { phire laser output pow- } \\
\text { er- } 200 \mathrm{~mW} \text {, inter-groove } \\
\text { distance } 60 \mu \mathrm{m}\end{array}$ & $\begin{array}{l}\text { Transbond } \\
\text { XT primer + } \\
\text { Transbond } \\
\text { XT light } \\
\text { cure com- } \\
\text { posite }\end{array}$ & $\begin{array}{l}\text { Maxillary } \\
\text { incisor } \\
\text { metal } \\
\text { brackets }\end{array}$ & $\begin{array}{l}37^{\circ} \mathrm{C} \text { distilled } \\
\text { water for } \\
24 \text { hrs. }\end{array}$ & Not done & $\begin{array}{l}G 1: 3.87 \pm 0.77 \\
\text { G2: } 4.25 \pm 0.51 \\
\text { G3: } 5.92 \pm 1.12 \\
\text { G4: } 3.74 \pm 0.10 \\
\text { G5: } 3.91 \pm 0.53 \\
\text { G6: } 5.68 \pm 0.94\end{array}$ \\
\hline $\begin{array}{l}\text { Byeon } \\
\text { et al. }{ }^{14} \\
(2017)\end{array}$ & 130 & $\begin{array}{l}\text { G1) Polishing } \\
\text { G2) Sandblasting } \\
\text { G3) Sandblasting + Silane } \\
\text { primer } \\
\text { G4) Sandblasting + MDP } \\
\text { primer } \\
\text { G5) Sandblasting + MDP } \\
\text { containing silane primer }\end{array}$ & $\begin{array}{l}\text { Transbond } \\
\text { XT light } \\
\text { cured com- } \\
\text { posite }\end{array}$ & $\begin{array}{l}\text { Maxillary } \\
\text { central } \\
\text { incisor } \\
\text { metal } \\
\text { brackets }\end{array}$ & $\begin{array}{l}37 \pm 1^{\circ} \mathrm{C} \text { dis- } \\
\text { tilled water } \\
\text { for } 24 \text { hrs. }\end{array}$ & $\begin{array}{l}5,000 \text { cycles } \\
\text { between } \\
5^{\circ}-55^{\circ} \mathrm{C} \text { with } \\
30 \text { s dwelling } \\
\text { time }\end{array}$ & $\begin{array}{c}\text { Before } \\
\text { thermocycling: } \\
\text { G1: } 2.6 \pm 1.1 \\
\text { G2: } 4.98 \pm 1.28 \\
\text { G3: } 5.13 \pm 0.85 \\
\text { G4: } 11.92 \pm 1.51 \\
\text { G5: } 13.36 \pm 2.31 \\
\text { After } \\
\text { thermocycling: } \\
\text { G1: } 0.70 \pm 0.4 \\
\text { G2: } 0.8 \pm 0.3 \\
\text { G3: } 1.5 \pm 0.4 \\
\text { G4: } 5.4 \pm 3.5 \\
\text { G5: } 5.7 \pm 1.2\end{array}$ \\
\hline
\end{tabular}


Table 2 - continuation: Data extraction.

\begin{tabular}{|c|c|c|c|c|c|c|c|}
\hline Study & Sample & Surface treatment & Adhesive & Brackets & Storage & $\begin{array}{l}\text { Thermocy- } \\
\text { cling }\end{array}$ & $\begin{array}{l}\text { Bond strength } \\
(\mathrm{MPa})\end{array}$ \\
\hline $\begin{array}{c}\text { Gar- } \\
\text { cia-Sanz } \\
\text { et al. }{ }^{17} \\
(2017)\end{array}$ & 300 & $\begin{array}{l}\text { G1) No treatment } \\
\text { G2) Sandblasting } \\
\text { G3) Tribochemical silica } \\
\text { coating + Silane primer } \\
\text { G4) Femtosecond laser } \\
\text { (200 mW) } \\
\text { G5) Sandblasting + Femto- } \\
\text { second laser ( } 200 \mathrm{~mW})\end{array}$ & $\begin{array}{l}\text { Transbond } \\
\text { XT primer + } \\
\text { Transbond } \\
\text { XT light } \\
\text { cure com- } \\
\text { posite }\end{array}$ & $\begin{array}{l}\text { i) Maxil- } \\
\text { lary cen- } \\
\text { tral incisor } \\
\text { metal } \\
\text { brackets. } \\
\text { ii) Maxil- } \\
\text { lary cen- } \\
\text { tral incisor } \\
\text { ceramic } \\
\text { brackets. }\end{array}$ & $\begin{array}{l}\text { Not men- } \\
\text { tioned }\end{array}$ & $\begin{array}{l}\text { Not men- } \\
\text { tioned }\end{array}$ & $\begin{array}{c}\text { Metal brackets: } \\
\text { G1: } 4.23 \pm 0.89 \\
\text { G2: } 4.46 \pm 1.21 \\
\text { G3: } 5.99 \pm 1.86 \\
\text { G4: } 6.72 \pm 2.30 \\
\text { G5: } 7.22 \pm 2.73 \\
\text { Ceramic } \\
\text { brackets: } \\
\text { G1: } 20.06 \pm 2.34 \\
\text { G2: } 25.01 \pm 4.45 \\
\text { G3: } 21.62 \pm 6.48 \\
\text { G4: } 23.18 \pm 6.51 \\
\text { G5: } 29.22 \pm 8.20\end{array}$ \\
\hline $\begin{array}{l}\text { Mehmeti } \\
\text { et al. }{ }^{24} \\
(2017)\end{array}$ & 20 & $\begin{array}{l}37 \% \text { phosphoric acid for } \\
120 \mathrm{~s}\end{array}$ & $\begin{array}{l}\text { Transbond } \\
\text { XT primer + } \\
\text { Transbond } \\
\text { XT light } \\
\text { cure com- } \\
\text { posite }\end{array}$ & $\begin{array}{l}\text { G1: Metal } \\
\text { brackets } \\
\text { G2: Poly- } \\
\text { crystalline } \\
\text { ceramic } \\
\text { brackets }\end{array}$ & $\begin{array}{c}\text { Waterbath for } \\
24 \mathrm{hrs} \text {. }\end{array}$ & Not done & $\begin{array}{l}\text { G1: } 7.35 \pm 3.41 \\
\text { G2: } 4.66 \pm 1.78\end{array}$ \\
\hline $\begin{array}{l}\text { Lee } \\
\text { et al. }{ }^{28} \\
\text { (2017) }\end{array}$ & 50 & $\begin{array}{l}\text { G1) Sandblasting } \\
\text { G2) Sandblasting + Metal/ } \\
\text { Zirconia primer } \\
\text { G3) Sandblasting + } \\
\text { Z-Prime plus } \\
\text { G4) Sandblasting + Zirco- } \\
\text { nia liner } \\
\text { G5) Sandblasting + Scotch- } \\
\text { bond universal adhesive }\end{array}$ & $\begin{array}{l}\text { Transbond } \\
\text { XT primer + } \\
\text { Transbond } \\
\text { XT light } \\
\text { cured com- } \\
\text { posite }\end{array}$ & $\begin{array}{l}\text { Ceramic } \\
\text { brackets }\end{array}$ & $\begin{array}{l}37^{\circ} \mathrm{C} \text { distilled } \\
\text { water for } \\
24 \text { hrs. }\end{array}$ & $\begin{array}{c}2,000 \text { cycles } \\
\text { between } \\
5^{\circ}-55^{\circ} \mathrm{C} \text { for } \\
1 \text { minute }\end{array}$ & $\begin{array}{c}G 1: 1.07 \pm 0.81 \\
\text { G2: } 5.16 \pm 0.83 \\
\text { G3: } 10.47 \pm 2.89 \\
\text { G4: } 9.55 \pm 1.75 \\
\text { G5: } 13.85 \pm 1.48\end{array}$ \\
\hline $\begin{array}{l}\text { Kim } \\
\text { et al. }{ }^{19} \\
(2017)\end{array}$ & 124 & $\begin{array}{l}\mathrm{G} 1) \text { Sandblasting }\left(\mathrm{Al}_{2} \mathrm{O}_{3}\right)+ \\
\text { Silane primer } \\
\mathrm{G} 2) \text { Sandblasting }\left(\mathrm{Al}_{2} \mathrm{O}_{3}\right)+ \\
\text { Zirconia prime plus } \\
\mathrm{G} 3) \text { Sandblasting }\left(\mathrm{Al}_{2} \mathrm{O}_{3}\right)+ \\
\text { universal bonding resin } \\
\text { G4) Sandblasting }\left(\mathrm{SiO}_{2}\right)+ \\
\text { Silane primer } \\
\text { G5) Sandblasting }\left(\mathrm{SiO}_{2}\right)+ \\
\text { Zirconia prime plus } \\
\text { G6) Sandblasting }\left(\mathrm{SiO}_{2}\right)+ \\
\text { universal bonding resin }\end{array}$ & $\begin{array}{l}\text { Transbond } \\
\text { XT primer + } \\
\text { Transbond } \\
\text { XT light } \\
\text { cured com- } \\
\text { posite }\end{array}$ & $\begin{array}{l}\text { Mandibu- } \\
\text { lar anteri- } \\
\text { or metal } \\
\text { brackets }\end{array}$ & $\begin{array}{c}100 \% \text { relative } \\
\text { humidity for } 7 \\
\text { days }\end{array}$ & $\begin{array}{l}2,000 \text { cycles } \\
\text { between } \\
5^{\circ}-55^{\circ} \mathrm{C} \text { for } 1 \\
\text { minute with } \\
\text { 20s dwelling } \\
\text { time. }\end{array}$ & $\begin{array}{c}\text { Non-thermocy- } \\
\text { cled: } \\
\text { G1: } 11.4 \pm 5.8 \\
\text { G2: } 21.6 \pm 3.3 \\
\text { G3: } 22.9 \pm 6.5 \\
\text { G4: } 19.7 \pm 4.1 \\
\text { G5: } 20.5 \pm 5.4 \\
\text { G6: } 24.2 \pm 2.8 \\
\text { Thermocycled: } \\
\text { G1: } 13.7 \pm 5.0 \\
\text { G2: } 20 \pm 4.9 \\
\text { G3: } 22.5 \pm 6.9 \\
\text { G4: } 25 \pm 5 \\
\text { G5: } 24.1 \pm 3.5 \\
\text { G6: } 26.2 \pm 3.1\end{array}$ \\
\hline
\end{tabular}


Table 2 - continuation: Data extraction.

\begin{tabular}{|c|c|c|c|c|c|c|c|}
\hline Study & Sample & Surface treatment & Adhesive & Brackets & Storage & $\begin{array}{l}\text { Thermocy- } \\
\text { cling }\end{array}$ & $\begin{array}{l}\text { Bond strength } \\
\qquad(\mathrm{MPa})\end{array}$ \\
\hline $\begin{array}{l}\text { Kim } \\
\text { et al. }{ }^{20} \\
\text { (2017) }\end{array}$ & 160 & $\begin{array}{c}\text { G1) Sandblasting + ESPE- } \\
\text { sil } \\
\text { G2) Sandblasting + Alloy } \\
\text { primer } \\
\text { G3) Sandblasting + Clearfil } \\
\text { ceramic primer } \\
\text { G4) Sandblasting + Single } \\
\text { bond universal primer } \\
\text { G5) Tribochemical silica } \\
\text { coating + ESPE-sil } \\
\text { G6) Tribochemical silica } \\
\text { coating + Alloy primer } \\
\text { G7) Tribochemical silica } \\
\text { coating + Clearfil ceramic } \\
\text { primer } \\
\text { G8) Tribochemical silica } \\
\text { coating + Single bond uni- } \\
\text { versal primer }\end{array}$ & $\begin{array}{l}\text { Transbond } \\
\text { XT light } \\
\text { cured com- } \\
\text { posite }\end{array}$ & $\begin{array}{l}\text { Maxillary } \\
\text { central } \\
\text { incisor } \\
\text { metal } \\
\text { brackets }\end{array}$ & $\begin{array}{l}\text { Deionized } \\
\text { water } 37^{\circ} \mathrm{C} \\
\text { for } 24 \mathrm{~h}\end{array}$ & $\begin{array}{l}5000 \text { cycles } \\
\text { between } \\
5^{\circ}-55^{\circ} \mathrm{C} \text { with } \\
\text { 30s dwelling } \\
\quad \text { time }\end{array}$ & $\begin{array}{c}\text { Non-thermocy- } \\
\text { cled: } \\
\text { G1: } 6.6 \pm 2.6 \\
\text { G2: } 15.9 \pm 5.2 \\
\text { G3: } 13.1 \pm 3.4 \\
\text { G4: } 16.7 \pm 5.6 \\
\text { G5: } 7.9 \pm 3.4 \\
\text { G6: } 17 \pm 3.9 \\
\text { G7: } 15.9 \pm 5.7 \\
\text { G8: } 19.4 \pm 4.5 \\
\text { Thermocycled: } \\
\text { G1: } 5.2 \pm 1.4 \\
\text { G2: } 14.3 \pm 2.9 \\
\text { G3: } 12.9 \pm 4.3 \\
\text { G4: } 15.2 \pm 5.3 \\
\text { G5: } 5.8 \pm 1.6 \\
\text { G6: } 8.6 \pm 1.7 \\
\text { G7: } 14.8 \pm 3.9 \\
\text { G8: } 7.1 \pm 0.9\end{array}$ \\
\hline $\begin{array}{l}\text { Amer and } \\
\text { Rayyan }^{13} \\
(2018)\end{array}$ & 60 & $\begin{array}{l}\text { G1) No surface treatment } \\
\text { G2) Sandblasting } \\
\text { G3) Soflex disc }\end{array}$ & $\begin{array}{c}\text { i) Clearfil } \\
\text { ceramic } \\
\text { primer + } \\
\text { Panavia F } \\
2.0 \text { adhe- } \\
\text { sive resin } \\
\text { cement } \\
\text { ii) Rely X } \\
\text { U200 self } \\
\text { adhesive } \\
\text { resin ce- } \\
\text { ment }\end{array}$ & $\begin{array}{l}\text { Lower } \\
\text { second } \\
\text { premo- } \\
\text { lar metal } \\
\text { brackets }\end{array}$ & $\begin{array}{l}37^{\circ} \mathrm{C} \text { distilled } \\
\text { water for } \\
24 \text { hrs. }\end{array}$ & $\begin{array}{l}500 \text { cycles } \\
\text { between } \\
5^{\circ}-55^{\circ} \mathrm{C} \text { with } \\
\text { 30s dwelling } \\
\quad \text { time }\end{array}$ & $\begin{array}{c}\text { Clearfil + Pan- } \\
\text { via F 2.0: } \\
\text { G1: } 0 \\
\text { G2: } 20.8 \pm 4.8 \\
\text { G3: } 12.3 \pm 2.8 \\
\text { Rely X U200: } \\
\text { G1: } 0 \\
\text { G2: } 16.7 \pm 4.6 \\
\text { G3: } 11.6 \pm 3\end{array}$ \\
\hline $\begin{array}{c}\text { Ihsan } \\
\text { and Al- } \\
\text { Dabagh29 } \\
\text { (2017) }\end{array}$ & 40 & $\begin{array}{c}\text { G1) Z prime plus primer } \\
\mathrm{G} 2) \text { Sandblasting }\left(\mathrm{Al}_{2} \mathrm{O}_{3}\right)+ \\
\text { Z prime plus primer } \\
\text { G3) Nd:YAG laser } \\
(0.888 \mathrm{~W}, 5 \mathrm{~s} \text {.) + Z-prime } \\
\text { plus primer } \\
\text { G4) Nd:YAG laser } \\
\text { (0.444 W, 10s) + Z-prime } \\
\text { plus primer }\end{array}$ & $\begin{array}{l}\text { Light-cured } \\
\text { composite }\end{array}$ & $\begin{array}{l}\text { Central } \\
\text { incisor } \\
\text { sapphire } \\
\text { brackets }\end{array}$ & $\begin{array}{l}37^{\circ} \mathrm{C} \text { distilled } \\
\text { water for } \\
24 \text { hrs. }\end{array}$ & Not done & $\begin{array}{l}G 1: 11.08 \pm 1.96 \\
G 2: 22.29 \pm 1.18 \\
G 3: 30.25 \pm 2.31 \\
G 4: 30.67 \pm 2.33\end{array}$ \\
\hline $\begin{array}{l}\text { Hosseini } \\
\text { et al. }{ }^{18} \\
(2016)\end{array}$ & 72 & $\begin{array}{l}\text { G1) No treatment } \\
\text { G2) Er:YAG laser } 1.5 \mathrm{~W} \\
\text { G3) Er:YAG laser } 2.5 \mathrm{~W} \\
\text { G4) Er:YAG laser } 3 \mathrm{~W} \\
\text { G5) Sandblasting } \\
\text { G6) Silane primer }\end{array}$ & $\begin{array}{l}\text { Transbond } \\
\text { XT light } \\
\text { cure com- } \\
\text { posite }\end{array}$ & $\begin{array}{l}\text { Maxillary } \\
\text { incisor } \\
\text { metal } \\
\text { brackets }\end{array}$ & $\begin{array}{c}37^{\circ} \mathrm{C} \text { distilled } \\
\text { water for } 24 \\
\text { hrs. }\end{array}$ & $\begin{array}{l}500 \text { cycles } \\
\text { between } \\
5^{\circ}-55^{\circ} \mathrm{C} \text { with } \\
\text { 30s dwelling } \\
\text { time }\end{array}$ & $\begin{array}{l}G 1: 0.31 \pm 0.23 \\
\text { G2: } 0.51 \pm 0.14 \\
\text { G3: } 1.11 \pm 0.40 \\
\text { G4: } 3.32 \pm 1.52 \\
\text { G5: } 9.50 \pm 2.92 \\
\text { G6: } 3.88 \pm 2.20\end{array}$ \\
\hline
\end{tabular}


Table 2 - continuation: Data extraction.

\begin{tabular}{|c|c|c|c|c|c|c|c|}
\hline Study & Sample & Surface treatment & Adhesive & Brackets & Storage & $\begin{array}{l}\text { Thermocy- } \\
\text { cling }\end{array}$ & $\begin{array}{l}\text { Bond strength } \\
\qquad(\mathrm{MPa})\end{array}$ \\
\hline $\begin{array}{l}\text { Lee } \\
\text { et al. }{ }^{22} \\
\text { (2015) }\end{array}$ & 40 & $\begin{array}{c}\text { G1) Non-glazed + Sand- } \\
\text { blasting + Zirconia primer } \\
\text { G2) Glazing + Sandblasting } \\
\text { + etching + Zirconia primer } \\
\text { G3) Glazing + sandblast- } \\
\text { ing + etching + porcelain } \\
\text { primer } \\
\text { G4) Glazing + sandblasting } \\
\text { + etching + zirconia prim- } \\
\text { er + porcelain primer }\end{array}$ & $\begin{array}{l}\text { Transbond } \\
\text { XT light } \\
\text { cured com- } \\
\text { posite }\end{array}$ & $\begin{array}{c}\text { Mandibu- } \\
\text { lar incisor } \\
\text { metal } \\
\text { brackets }\end{array}$ & $\begin{array}{c}37^{\circ} \mathrm{C} \text { distilled } \\
\text { water }\end{array}$ & $\begin{array}{c}2,000 \text { cycles } \\
\text { between } \\
5^{\circ}-55^{\circ} \mathrm{C} \text { with } \\
30 \text { s dwelling } \\
\text { time }\end{array}$ & $\begin{array}{c}\text { G1: } 13.7 \pm 1.3 \\
\text { G2: } 3.7 \pm 0.9 \\
\text { G3: } 16 \pm 2.6 \\
\text { G4: } 14.4 \pm 1.7\end{array}$ \\
\hline $\begin{array}{l}\text { Kwak } \\
\text { et al. }{ }^{21} \\
(2016)\end{array}$ & 70 & $\begin{array}{c}\mathrm{G} 1 \text { - unglazed): Silicon car- } \\
\text { bide paper roughening } \\
\text { G2) Diamond bur +Z-prime } \\
\text { plus primer } \\
\text { G3) Pumice + Monobond-S } \\
\text { primer } \\
\text { G4) } 4 \% \text { hydrofluoric acid + } \\
\text { Monobond-S primer } \\
\text { G5) Sandblasting }\left(\mathrm{Al}_{2} \mathrm{O}_{3}\right)+ \\
\text { Monobond-S primer } \\
\text { G6) Sandblasting }\left(\mathrm{Al}_{2} \mathrm{O}_{3}\right)+ \\
\text { Z-prime plus primer } \\
\text { G7) Sandblasting }\left(\mathrm{SiO}_{2}\right)+ \\
\text { Monobond-S primer }\end{array}$ & $\begin{array}{l}\text { Transbond } \\
\text { XT light } \\
\text { cure com- } \\
\text { posite resin }\end{array}$ & $\begin{array}{c}\text { Mandibu- } \\
\text { lar incisor } \\
\text { metal } \\
\text { brackets }\end{array}$ & $\begin{array}{c}37^{\circ} \mathrm{C} \text { water for } \\
24 \text { hrs }\end{array}$ & $\begin{array}{c}1,000 \text { cycles } \\
\text { between } \\
5^{\circ}-55^{\circ} \mathrm{C}\end{array}$ & $\begin{array}{l}\text { G1: } 13.38 \pm 2.57 \\
\text { G2: } 15.48 \pm 3.15 \\
\text { G3: } 14.90 \pm 2.75 \\
\text { G4: } 15.24 \pm 3.36 \\
\text { G5: } 15.78 \pm 2.39 \\
\text { G6: } 4.60 \pm 1.08 \\
\text { G7: } 14.81 \pm 2.91\end{array}$ \\
\hline $\begin{array}{l}\text { Yassaei } \\
\text { et al. }{ }^{25} \\
(2015)\end{array}$ & 72 & $\begin{array}{l}\text { G1) 9.6\% hydrofluoric acid } \\
+ \text { silane primer } \\
\text { G2) Sandblasting }\left(\mathrm{Al}_{2} \mathrm{O}_{3}\right)+ \\
\text { Silane primer } \\
\text { G3) Er:YAG laser }(1 \mathrm{~W})+ \\
\text { Silane primer } \\
\text { G4) Er:YAG laser }(2 \mathrm{~W})+ \\
\text { Silane primer }\end{array}$ & $\begin{array}{l}\text { Light-cured } \\
\text { composite } \\
\text { resin }\end{array}$ & $\begin{array}{l}\text { Metallic } \\
\text { maxillary } \\
\text { central } \\
\text { incisor } \\
\text { brackets }\end{array}$ & $\begin{array}{c}37^{\circ} \mathrm{C} \text { water for } \\
24 \text { hrs }\end{array}$ & $\begin{array}{c}500 \text { cycles } \\
\text { between } \\
5^{\circ}-55^{\circ} \mathrm{C} \text { with } \\
\text { 30s dwelling } \\
\text { time }\end{array}$ & $\begin{array}{l}\text { G1: } 5.8 \pm 0.78 \\
\text { G2: } 7.8 \pm 1.02 \\
\text { G3: } 6.8 \pm 0.92 \\
\text { G4: } 6.9 \pm 1.13\end{array}$ \\
\hline
\end{tabular}




\section{RISK OF BIAS}

The methodological merits of the selected studies were assessed by both reviewers individually. The assessment tool was adapted from previous in-vitro systematic reviews and meta-analyses, judging on the following parameters: sample size calculation, presence of control group, use of materials according to the manufacturer's instruction, standardized sample preparation, surface treatment and bonding done by the same operator, adequate description of thermocycling and the appropriate statistical analysis (i.e., reporting mean, standard deviation and $p$-values). ${ }^{9,10}$ Any of these parameters reported by the articles was ticked with " $Y$ " (yes) for the particular section. In case any parameter was missing, was marked with an "N" (no). Articles reporting only one (1) to three (3) of the items were considered as having a high risk of bias; four (4) to five (5) items, as medium risk of bias; and six (6) to seven (7) items, as low risk of bias.9,10 Again, the interexaminer agreement was analyzed by Cohen's kappa statistics, and the third reviewer's opinion was requested in any event of disagreement.

\section{RESULTS}

\section{LITERATURE SEARCH}

A total of 109 studies was identified (Table 1). After the removal of the duplicates, 91 articles remained. Upon careful screening of the titles and abstracts, 48 articles were further excluded. 
The kappa value for interexaminers agreement was $k=0.816$ $(p<0.001)$. Forty three full-text articles were screened thoroughly for eligibility and 24 articles were excluded for valid reasons (Supplementary Table 1 ). Finally, 19 articles were selected for the review. The PRISMA flow diagram of the study selection procedure is presented in Figure 1.

Supplementary table 1: Reason for exclusion.

\begin{tabular}{c|c} 
Article & Reason for exclusion \\
\hline Ahluwalia et al., 2013 & Zirconium surface was not included \\
\hline Buyuk et al., 2018 & Zirconium surface was not included \\
\hline Byeon et al., 2016 & Full-text article was not found \\
\hline Di guida et al., 2019 & Zirconium surface was not included \\
\hline Dong et al., 2016 & Full-text article was not found \\
\hline Elsaka et al., 2016 & Zirconium surface was not included \\
\hline Juntavee et al., 2018 & Zirconium surface was not included \\
\hline Kawai et al., 2011 & Orthodontic brackets were not included \\
\hline Kaya et al., 2019 & Zirconium surface was not included \\
\hline Murthy et al., 2014 & Orthodontic brackets were not included \\
\hline Soliman et al., 2018 & Zirconium surface was not included \\
\hline Zhang et al., 2016 & Zirconium surface was not included \\
\hline Pitriani et al., 2019 & Zirconium surface not included \\
\hline Gardiner et al., 2019 & Orthodontic buccal tubes included, not brackets \\
\hline Mehta et al., 2016 & Shear bond strength not measured \\
\hline Mirhashmi et al., 2020 & Shear bond strength in MPa units not measured \\
\hline Abuelenain et al., 2020 & Lithium silicate reinforced with zirconia \\
\hline Ahluwalia et al., 2013 & Porcelain fused to zirconia crowns \\
\hline Amer and Rayyan, 2018 & Glazed monolithic zirconia crowns \\
\hline Bilgic et al., 2013 & Porcelain fused to zirconia crowns \\
\hline Franz et al., 2019 & Zirconium oxide sinter ceramics \\
\hline Ismail and Sheikh, 2018 & Porcelain veneered zirconia crown \\
\hline Oldham et al., 2019 & Lithium silicate infused with zirconia \\
\hline Pouyanfar et al., 2019 & Y-TZP zirconia was not included \\
\hline
\end{tabular}




\section{PRISMA 2009 Flow Diagram}

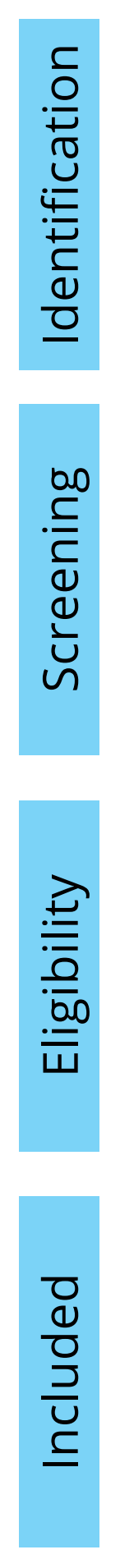

Records identified through database searching $(n=22)$

Additional records identified through other sources $(n=87)$
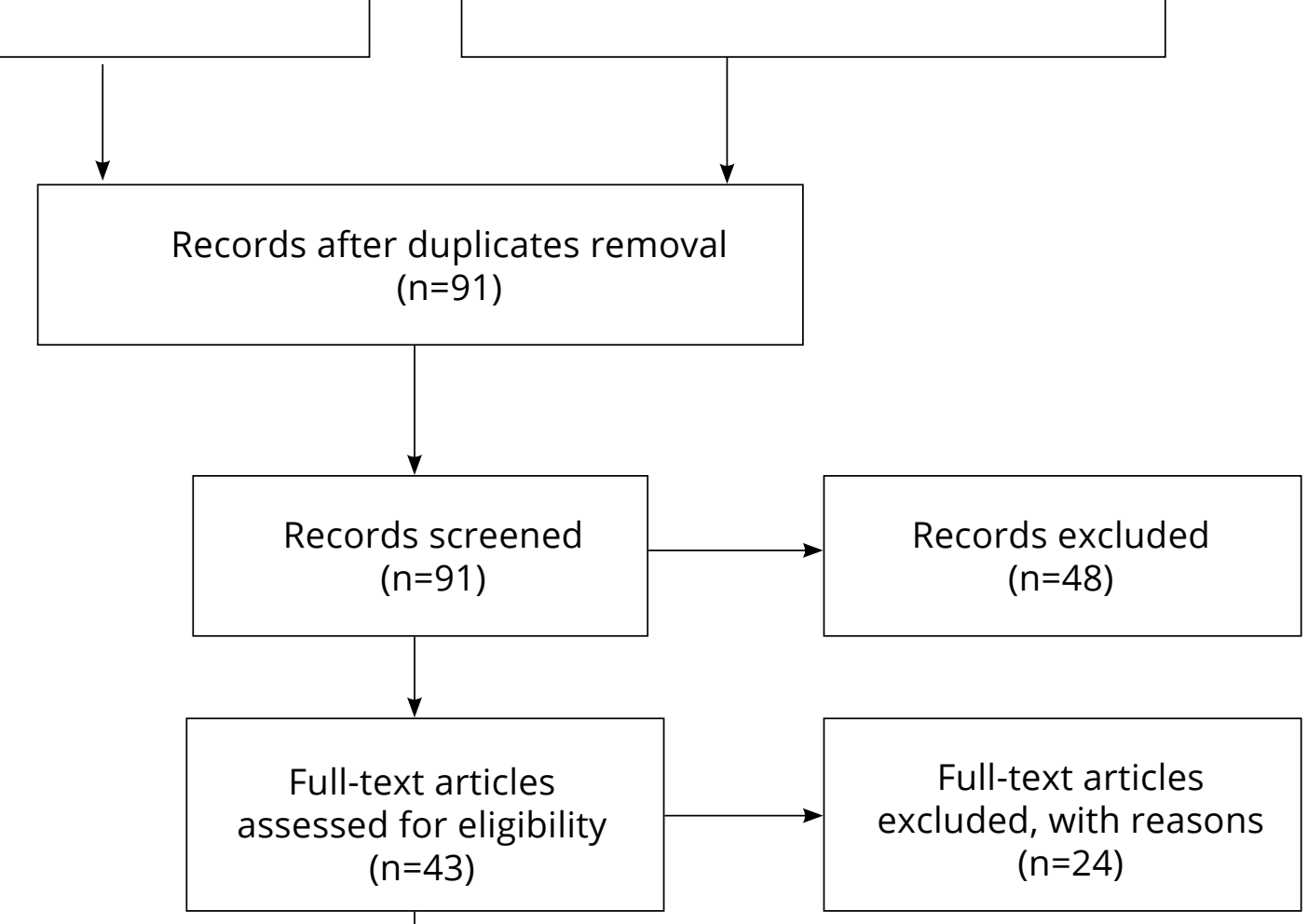

Studies included in

qualitative synthesis $(n=19)$

Figure 1: Flow diagram of the study selection strategy. 


\section{QUALITATIVE ASSESSMENT}

For the qualitative analysis, the inter-reviewers reliability of the extracted data was confirmed $(\mathrm{k}=0.89, p<0.001)$. Three types of brackets were studied. Metallic brackets were the most common; ${ }^{11-24}$ followed by the ceramic ${ }^{2,4,15,17,23,24,26,27}$, and sapphire brackets. ${ }^{28}$ Both mechanical and chemical methods of surface treatment were identified on the Y-TZP zirconia surface. Mechanical means included lasers, sandblasting, polishing and soflex disc, silicon carbide paper, and diamond bur roughening.,24,12-21,25,26,28 Chemically, acid etching (9.6\% hydrofluoric acid, 37\% phosphoric acid), application of primers, and universal adhesive were found. 2,12,14,16-20,22,25,27,28 Sandblasting was the most found surface treatment. Two types of sandblasting were noticed: conventional $\left(\mathrm{Al}_{2} \mathrm{O}_{3}\right)$ sandblasting and tribochemical silica $\left(\mathrm{SiO}_{2}\right)$ coated sandblasting. ${ }^{17,19-21}$ Sandblasting method differed between the studies as the variables like particle size $(25 \mu \mathrm{m}-110 \mu \mathrm{m})$, pressure (0.14 MPa-0.4 MPa), time (5 seconds-20seconds), and the distance (10mm-20mm) were not homogeneous. Three types of lasers were utilized: erbium-doped yttrium aluminum garnet laser (Er:YAG), neodymium-doped yttrium aluminum garnet laser (Nd:YAG), and the femtosecond laser. 12,15-18,25,28 Sandblastings and lasers were also combined with various primers and universal adhesive. 2,14,15,19,20,22,25,27,28 Eight studies treated Y-TZP surface with sandblasting only; $4,12,14,16-18,27$ five studies combined sandblasting with silane primer, ${ }^{14,17,19,20,25}$ five studies combined sandblasting with MDP (methacryloyloxydecyl dihydrogen phosphate) primers; ${ }^{14,19,20,27,28}$ four studies combined 
universal adhesive; ${ }^{2,19,20,27}$ three studies combined MDP containing silane primer, 2,14,20 and two studies combined zirconia primer.22,27 Lasers were used alone in four studies; ${ }^{12,16-18}$ and as an adjunct with MDP primer and silane primer in one study each. ${ }^{18,25}$ One study also combined femtosecond laser and sandblasting to treat Y-TZP surface. ${ }^{17}$ Variability was observed in laser settings like power output (40 $\mathrm{mW}$ to $3 \mathrm{~W})$; mean energy settings $(50 \mathrm{~mJ}$ to $300 \mathrm{~mJ})$, distance $(60 \mu \mathrm{m}$ to $10 \mathrm{~mm})$, and the application time (5 seconds to 2 minutes). Except for two studies, ${ }^{18,28}$ no study was found to treat the Y-TZP surface solely with primers before orthodontic bonding. The trade names and chemical composition of the primers used to treat Y-TZP surface are listed in Table 3.

Table 3: Primers used to treat Y-TZP surface.

\begin{tabular}{|c|c|c|}
\hline Primers & Trade name & Chemical composition \\
\hline Silane primer & $\begin{array}{l}\text { ESPE Sil, Rely X, Reliance porcelain } \\
\text { conditioner }\end{array}$ & 3-TMSPMA, Ethanol \\
\hline \multirow{3}{*}{ MDP Primers } & Z-PRIME Plus & MDP, Ethanol \\
\hline & Zirconia liner & MMA, 10-MDP, 4-methoxyphenol (HQME) \\
\hline & Primer Alloy & $\begin{array}{l}\text { 6-(4-vinylbenzyl-n-propyl) amino-1,3,5-tri- } \\
\text { azine-2,4 dithiol (VBATDT), 10-MDP, acetone }\end{array}$ \\
\hline MDP containing Silane primer & Clearfil Ceramic Primer & 10-MDP, 3-TMSPMA, Ethanol. \\
\hline Universal adhesive & $\begin{array}{c}\text { Clearfil S3 Bond, Scotchbond uni- } \\
\text { versal adhesive, Single Bond Uni- } \\
\text { versal }\end{array}$ & $\begin{array}{l}\text { 10-MDP, bis-GMA, HEMA, hydrophobic de- } \\
\text { methacrylate, dl-camphorquinone, ethyl alco- } \\
\text { hol, water, silanated colloidal silica }\end{array}$ \\
\hline Orthodontic primer & Transbond XT adhesive primer & $\begin{array}{l}\text { TEGMA, bis-GMA, triphenylantimony, 4-(dime- } \\
\text { thylamino)-benzeneethanol, dl-camphorquinone, } \\
\text { ethyl alcohol, water, silanated colloidal silica }\end{array}$ \\
\hline Zirconia primer & Metal/Zirconia primer & $\begin{array}{l}\text { Tertiary butyl alcohol, methyl isobutyl ketone, } \\
\text { phosphoric acid acrylate, benzoylperoxide. }\end{array}$ \\
\hline
\end{tabular}

Abbreviations: 3-TMSPMA (3-trimethoxysilylpropyl methacrylate), 10-MDP (10-methacryloyloxydecyl dihydrogen phosphate), Bis-GMA (bisphenol-A-diglycidyl methacrylate), HEMA (hydroxyethyl methacrylate), TEGMA (triethylene glycol dimethacrylate), MMA (methyl methacrylate), HQME (hydroquinone monoethyl ether). 
Two studies did not thermocycled their samples. ${ }^{16,17}$ Different protocols of thermocycling were observed between the studies; mostly at the range of $500-10,000$ cycles, at $5-55^{\circ} \mathrm{C}$ temperature with dwell time of 20 to 30 seconds. The $37^{\circ} \mathrm{C}$ distilled water for 24 hours was the most common method of storage before bonding. ${ }^{13,14,16,18,21,22,25-28}$ Transbond $\mathrm{XT}^{\odot}$ primer and light-curing composite were widely used as an adhesive. $2,9,12,14,17-20,22,27$

Qualitative analyses of the included studies are detailed in Table 2.

\section{RISK OF BIAS}

Based on the criteria applied for the quality assessment of the selected studies; thirteen (13) studies scored medium risk of bias, three (3) studies scored low risk of bias, and the remaining three (3) studies scored high risk of bias. Sample size calculation was surprisingly absent (except for one selected study). Half of the studies had no control groups and surface treatment. Bonding was done by the same operator in only $47.4 \%$ of cases. The frequency of the rest of the parameters was sufficient (Fig 2). 
Sample size calculation

Control group

Materials used according to the manufacturer's instruction

Standardized sample preparation

Surface treatment and bonding done by the same operator

Adequate description of thermocycling

Appropriate statistical analysis
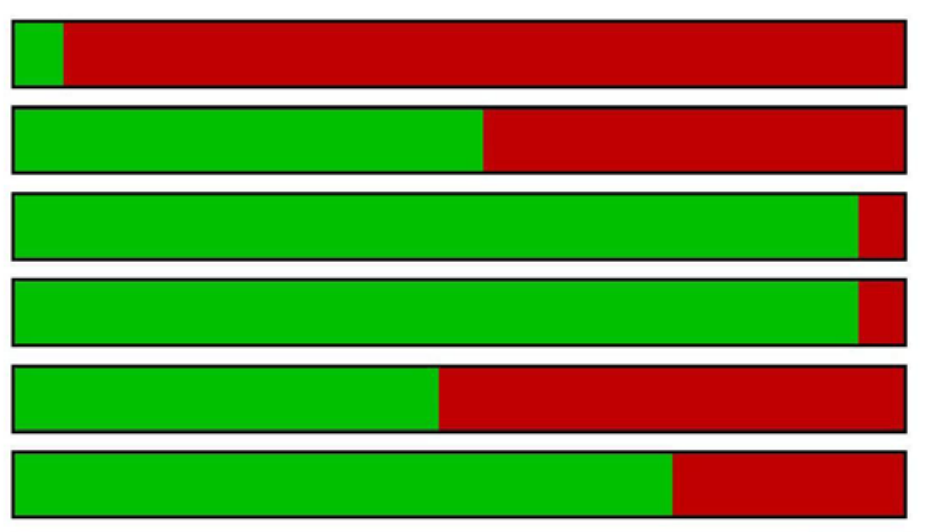

\begin{tabular}{|c|c|c|}
\hline $0 \%$ & $25 \%$ & $50 \%$ \\
\hline
\end{tabular}

Yes (low risk)

Unclear

No (high risk)

\begin{tabular}{|c|c|c|c|c|c|c|c|c|c|c|c|c|c|c|c|c|c|c|c|}
\hline 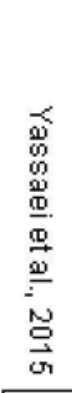 & 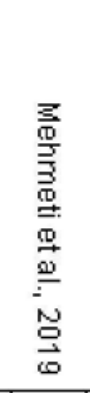 & 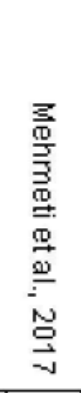 & 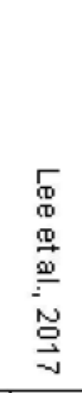 & 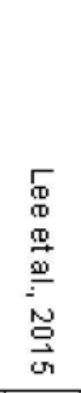 & 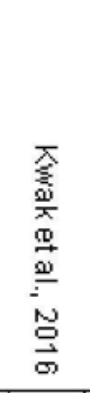 & 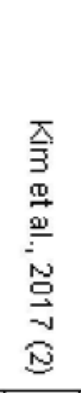 & 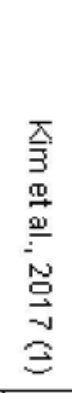 & 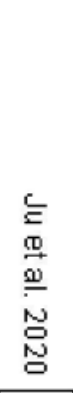 & 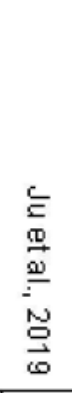 & 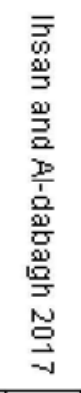 & 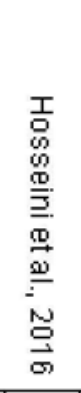 & 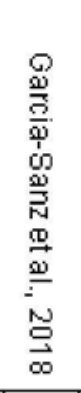 & 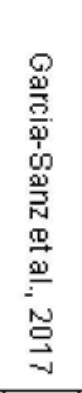 & 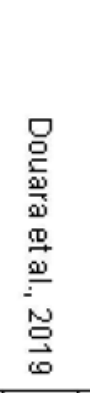 & 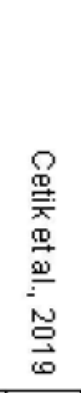 & 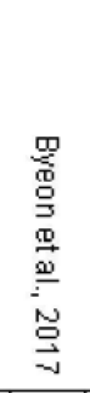 & 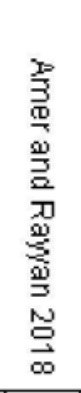 & 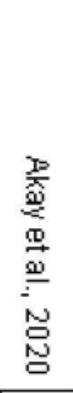 & \\
\hline (1) & (1) & (1) & O & ? & (1) & (1) & (1) & (1) & (1) & (1) & (1) & (1) & (1) & $\odot$ & (1) & (1) & (1) & (1) & Sample size calculation \\
\hline (1) & (1) & (1) & $\odot$ & O & $\odot$ & (1) & (1) & $\odot$ & $\odot$ & + & $\odot$ & $\odot$ & $\odot$ & (1) & (1) & $\odot$ & + & (1) & Control group \\
\hline (1) & + & $\odot$ & $\odot$ & $\odot$ & $\odot$ & + & $\odot$ & $\odot$ & $\odot$ & + & $\odot$ & $\odot$ & $\odot$ & + & $\odot$ & + & $\odot$ & + & Materials used according to the manufacturer's instruction \\
\hline+ & $\odot$ & $\odot$ & $\odot$ & $\odot$ & $\odot$ & $\odot$ & $\odot$ & (1) & $\odot$ & $\odot$ & $\odot$ & $\odot$ & + & + & $\odot$ & $\odot$ & + & $\odot$ & Standardized sample preparation \\
\hline (1) & + & + & $\odot$ & (1) & + & 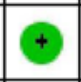 & (1) & (1) & $\odot$ & (1) & (1) & + & + & (1) & (1) & (1) & (1) & + & Surface treatment and bonding done by the same operator \\
\hline$\odot$ & $\odot$ & (1) & $\odot$ & $\odot$ & $\odot$ & 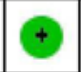 & $\odot$ & $\odot$ & $\odot$ & (1) & $\odot$ & (1) & (1) & $\odot$ & (1) & $\odot$ & + & $\odot$ & Adequate description of thermocycling \\
\hline+ & $\odot$ & + & $\odot$ & + & + & + & + & (1) & + & + & + & (1) & + & + & + & + & + & + & Appropriate statistical analysis \\
\hline
\end{tabular}

Figure 2: Summary of the risk of bias of the included studies, 


\section{DISCUSSION}

In the examined literature, different surface treatment protocols have been studied extensively regarding the adherence between the orthodontic brackets and the Y-TZP zirconia ceramics. Previously, several reviews on the ceramic surface conditioning and other testing parameters were published either with or

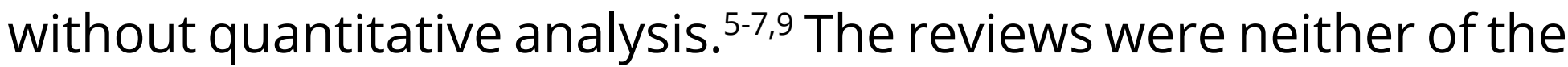
specific types of ceramic surface nor on the effect of orthodontic brackets. Regarding mechanical testing protocols, the shear bond strength (SBS) test is the most popular method because it is less complicated and produces results with a smaller standard deviation. ${ }^{5}$ Therefore, the effect of surface treatment methods on the SBS of the orthodontic brackets, particularly on the Y-TZP zirconia ceramic surface, was reviewed.

In this review, 19 studies were included for in-depth analysis testing SBS of orthodontic brackets to Y-TZP surface bonded with light-cure resin/composites. Meta-analysis could not be performed due to the high heterogeneity of the included studies, limiting the study outcome. This high variability may be due to the variability in sample size, storage, thermocycling, sandblasting, different laser settings, bracket materials (i.e. metal, ceramic), bracket anatomy, and base design. Maxillary central incisor metallic bracket was mostly studied. ${ }^{12,14,16-18,20,25}$ On reviewing the studies that compared the 
SBS between the metallic and the ceramic brackets, mixed outcomes were observed. ${ }^{15,17,26}$ A previously published research found that the ceramic brackets had greater bond strength to the Y-TZP surface than the metal brackets. ${ }^{17}$ On 37\% phosphoric acid etching, metallic brackets exhibited significantly better SBS than ceramic brackets. ${ }^{24}$ In a study, $37 \%$ phosphoric acid was combined with silane primer to enhance the SBS of the ceramic brackets; but the result was not significant. ${ }^{26}$ In comparison to phosphoric acid etching, the hydrofluoric acid etching did not improve the SBS in both metallic and ceramic brackets. ${ }^{26}$ In fact, surface pretreatment with hydrofluoric acid resulted in lower SBS of orthodontic brackets, in comparison to sandblasting, Er:YAG laser irradiation, and 37\% phosphoric acid etching. ${ }^{12,26}$ Due to the possibility of weakening the ceramic surface, the use of hydrofluoric acid was condemned. ${ }^{26}$ Both metallic and ceramic brackets exhibited higher SBS to the sandblasted and laser-irradiated surfaces in different studies. One study reported higher SBS of ceramic brackets on sandblasted, tribochemical silica-coated and femtosecond laser irradiated surfaces. ${ }^{17}$ Conversely, another study reported higher SBS of metallic brackets on the sandblasted and the Er:YAG laser-irradiated surface. ${ }^{15}$ Surprisingly, comparative analysis of the SBS of metal and ceramic brackets on Y-TZP surface treated by various primers could not be found. 
Sandblasting was found as the most common surface treatment method among the included studies. It promotes mechanical bonding by creating surface irregularities through air abrasion, using either alumina or silica-coated alumina particles. The latter procedure exhibited greater SBS in many studies. ${ }^{12,19-21}$ The CoJet ${ }^{\mathrm{TM}}$ system that applies silica-coated alumina particles not only abrades the ceramic surface, but also creates silica enriched outer surface favorable for silanization. ${ }^{6}$ Surface irregularities created by both of these particles were similar in previous studies. ${ }^{17,20}$ However, the surface free energy was greater in silica-coated sandblasting. ${ }^{19,20}$ Therefore, the additional application of primers promoting chemical adhesion might have contributed to the higher SBS. Following both forms of sandblasting, primers like silane, MDP, MDP containing silane primers or single-step universal adhesives were applied to optimize the SBS. 2,4,19,20,22 The primers and universal adhesives that contain MDP are capable of chemically bond to zirconia. The bonding occurs between the phosphate ester of MDP and the hydroxyl groups over the zirconia surface. ${ }^{14}$ The silane primers had lower SBS in comparison to the universal bonding resin, MDP, or MDP containing silane primers, as they do not form a chemical bond with zirconia unless the surface is coated with silica. ${ }^{14,19,20}$ 
Mechanical surface treatments such as sandblasting and lasers also have been studied. ${ }^{12,16-18,25,29}$ Lasers produce surface roughness by a process called ablation, which involves micro explosions and vaporization. ${ }^{6}$ Both Er:YAG and Nd:YAG laser irradiation of zirconia surface resulted in inferior SBS, compared to sandblasting. ${ }^{12,15,18,25}$ The Er:YAG lasers alone failed to achieve Reynolds's optimal SBS range of 5.9 - 7.8 MPa. 12,18,30 Yet, in combination with silane primer, the SBS was raised to 6.9 $\mathrm{MPa}{ }^{25}$ The additional chemical reaction and wetting ability of the silane may have contributed to this finding. Besides, at high power output (above $200 \mathrm{~mJ}$ ) the laser generates high heat, which can be detrimental to the surrounding zirconia surface. ${ }^{31}$ The femtosecond lasers, at $200 \mathrm{~mW}$ power output and $60 \mu \mathrm{m}$ inter-groove distance, generated SBS (5.68 MPa) closer to the optimal level. ${ }^{15}$ In separate studies, the femtosecond laser-treated zirconia surface had better SBS compared to both alumina $\left(\mathrm{Al}_{2} \mathrm{O}_{3}\right)$ and silica $\left(\mathrm{SiO}_{2}\right)$ coated sandblasting. ${ }^{16,17}$ Better SBS was achieved when the femtosecond laser was combined with sandblasting, but not significantly greater than the femtosecond laser alone. ${ }^{17}$ Thereby, additional surface preparation as an adjunct to femtosecond laser can be avoided to save time, cost and patient discomfort. Besides, the laser has no reports of thermal damage due to surface irradiation. ${ }^{32}$ 
Following thermocycling, there was a noticeable reduction in SBS irrespective of the surface pre-treatment methods. 2,14,19,20 Biodegradation of the treated Y-TZP surface, bonded brackets, and adhesives may have contributed to this evidence. Hence, the assessment of the bond strength in a simulated clinical environment (i.e., in-vivo experiment) is necessary. Thermocycling is an artificial aging procedure to test the longterm effect of bond strength. According to a study, 10,000 cycles of thermocycling are equivalent to one year of usage in the oral cavity. ${ }^{33}$ Application of MDP and MDP containing silane primers resulted in durable and optimal SBS on both forms of sandblasted Y-TZP, even after 10,000 cycles of thermocycling. ${ }^{2,20}$ Conversely, in the case of universal adhesives, the SBS was stable up to 2,000 cycles, but degraded significantly after 10,000 cycles. $^{2,20,27}$ Among lasers, the effect of thermocycling on the Er:YAG and Nd:YAG laser irradiated surfaces were tested. The SBS on Er:YAG laser-treated surface was found to be just clinically acceptable within the range of 500-2,000 cycles. ${ }^{12,25}$ In the case of $\mathrm{Nd}$ :YAG laser, the SBS was below an acceptable level after thermocycling. ${ }^{12}$ Surprisingly, the effect of thermocycling on the femtosecond laser irradiation could not be found. 
The validation risk of the bias tool utilized in this study could not be confirmed. This fact should be regarded as an important limitation of the study, but the contents of this quality assessment tool seemed to be more justifiable and relevant to the methodology of the selected studies. The absence of meta-analysis is another limitation reflecting the heterogeneity of the studies.

\section{CONCLUSION}

As this review is solely based on the qualitative analysis of the laboratory-based in-vitro findings, the results should be interpreted with caution. To answer the research question of this review more precisely, quantitative analysis is deemed necessary. Therefore, standardization of the study protocol is necessary. However, certain points may be advised, in light of this comprehensive review:

a) Hydrofluoric acid etching can be avoided to treat the Y-TZP surface, as it did not remarkably improve the SBS, considering the damage to the ceramic surface. 
b) Mechanical pretreatments like sandblasting and lasers are useful, as they both improved the SBS of orthodontic brackets.

c) Tribochemical silica-coated sandblasting with the advantage of chemical adhesion resulted in greater SBS than conventional sandblasting.

d) Among lasers, the femtosecond laser can be suggested as the first choice; although the effect of thermocycling on this laser irradiated zirconia surface is unknown.

e) Use of primers, particularly the MDP and the MDP containing silane primers as an adjunct to the mechanical pretreatments may be justified. There is a concern over the longevity of the universal adhesive. 
AUTHORS' CONTRIBUTIONS

Tamzid Ahmed (TA)

Nashid Fareen (NF)

Mohammad Khursheed Alam (MKA)
Conception or design of the study:

TA, NF, MKA.

Data acquisition, analysis or

interpretation:

TA, NF, MKA.

Writing the article:

TA, NF.

Critical revision of the article:

TA, NF, MKA.

Final approval of the article:

TA, NF, MKA.

Overall responsibility:

TA, MKA.

The authors report no commercial, proprietary or financial interest in the products or companies described in this article. 


\section{REFERENCES}

1. Heintze SD, Rousson V. Survival of zirconia- and metal-supported fixed dental prostheses: a systematic review. Int J Prosthodont. 2010 Nov-Dec;23(6):493-502.

2. Ju GY, Oh S, Lim BS, Lee HS, Chung SH. Effect of simplified bonding on shear bond strength between ceramic brackets and dental zirconia. Materials (Basel). 2019 May 20;12(10):1640.

3. Piconi C, Maccauro G. Zirconia as a ceramic biomaterial. Biomaterials. 1999 Jan;20(1):1-25.

4. Ju GY, Lim BS, Moon W, Park SY, Oh S, Chung SH. Primer-treated ceramic bracket increases shear bond strength on dental zirconia surface. Materials (Basel). 2020 Sep 16;13(18):4106.

5. Inokoshi M, De Munck J, Minakuchi S, Van Meerbeek B. Metaanalysis of bonding effectiveness to zirconia ceramics. J Dent Res. 2014 Apr;93(4):329-34.

6. Mattiello RDL, Coelho TMK, Insaurralde E, Coelho AAK, Terra GP, Kasuya AVB, et al. A review of surface treatment methods to improve the adhesive cementation of zirconia-based ceramics. Int Sch Res Notices. 2013;2013:1-10.

7. Papia E, Larsson C, du Toit M, Vult von Steyern P. Bonding between oxide ceramics and adhesive cement systems: a systematic review. J Biomed Mater Res B Appl Biomater. 2014 Feb;102(2):395-413. 
8. Cohen J. A coefficient of agreement for nominal scales. Educ Psychol Meas. 1960;20(1):37-46.

9. García-Sanz V, Paredes-Gallardo V, Mendoza-Yero O, CarbonellLeal M, Albaladejo A, Montiel-Company JM, et al. The effects of lasers on bond strength to ceramic materials: a systematic review and meta-analysis. PLoS One. 2018 Jan 2;13(1):e0190736.

10. Sarkis-Onofre R, Skupien JA, Cenci MS, Moraes RR, Pereira-Cenci $T$. The role of resin cement on bond strength of glass-fiber posts luted into root canals: a systematic review and meta-analysis of in vitro studies. Oper Dent. 2014 Jan-Feb;39(1):E31-44.

11. Higgins JP, Thomas J, Chandler J, Cumpston M, Li T, Page MJ, editors. Cochrane handbook for systematic reviews of interventions. 2nd ed. Chichester: John Wiley \& Sons; 2019.

12. Akay C, Okşayan R, Özdemir H. Influence of various types of surface modifications on the shear bond strength of orthodontic brackets on Y-TZP zirconia ceramics. J Aust Ceram Soc. 2020 Jun:1-5.

13. Amer JY, Rayyan MM. Effect of different surface treatments and bonding modalities on the shear bond strength between metallic orthodontic brackets and glazed monolithic zirconia crowns. J Orthod Sci. 2018 Nov 15;7:23.

14. Byeon SM, Lee MH, Bae TS. Shear bond strength of $\mathrm{Al}_{2} \mathrm{O}_{3}$ sandblasted Y-TZP ceramic to the orthodontic metal bracket. Materials (Basel). 2017 Feb 9;10(2):148. 
15. Cetik S, Ha TH, Sitri L, Duterme H, Pham V, Atash R. Comparison of shear strength of metal and ceramic orthodontic brackets cemented to zirconia depending on surface treatment: an in vitro study. Eur J Dent. 2019 May;13(2):150-5.

16. García-Sanz V, Paredes-Gallardo V, Bellot-Arcís C, Martínez-León L, Torres-Mendieta R, Montero J, et al. Femtosecond laser settings for optimal bracket bonding to zirconia. Lasers Med Sci. 2019 Mar;34(2):297-304.

17. García-Sanz V, Paredes-Gallardo V, Bellot-Arcís C, Mendoza-Yero O, Doñate-Buendía C, Montero J, et al. Effects of femtosecond laser and other surface treatments on the bond strength of metallic and ceramic orthodontic brackets to zirconia. PLoS One. 2017 Oct 19;12(10):e0186796.

18. Hosseini MH, Gorjizadeh F, Etemadi A, Baghaeian P. Shear Bond Strength of Metal brackets to Zirconia Treated with Er:YAG Laser Sandblasting and Silane. J Islam Dent Assoc Iran. 2016;28(3):1107.

19. Kim J, Park C, Lee JS, Ahn J, Lee Y. The effect of various types of mechanical and chemical preconditioning on the shear bond strength of orthodontic brackets on zirconia restorations. Scanning. 2017 Jan 11;2017:6243179.

20. Kim NH, Kim YJ, Lee DY. Bond strengths of orthodontic metal brackets to tribochemically silica-coated zirconia surfaces using different 10-methacryloyloxydecyl dihydrogen phosphatecontaining primers. J Adhes Dent. 2017;19(1):21-9. 
21. Kwak JY, Jung HK, Choi IK, Kwon TY. Orthodontic bracket bonding to glazed full-contour zirconia. Restor Dent Endod. 2016 May;41(2):106-13.

22. Lee JH, Lee M, Kim KN, Hwang CJ. Resin bonding of metal brackets to glazed zirconia with a porcelain primer. Korean J Orthod. 2015 Nov;45(6):299-307.

23. Mehmeti B. Evaluation of the shear bond strength of orthodontic brackets bonded to different ceramic surfaces [tese]. Zagreb: University of Zagreb; 2018.

24. Mehmeti B, Azizi B, Kelmendi J, Iljazi-Shahiqi D, Alar Ž, AnićMilošević S. Shear Bond Strength of Orthodontic Brackets Bonded to Zirconium Crowns. Acta Stomatol Croat. 2017 Jun;51(2):99-105.

25. Yassaei S, Aghili HA, Davari A, Mostafavi SM. Effect of four methods of surface treatment on shear bond strength of orthodontic brackets to zirconium. J Dent (Tehran). 2015 Apr;12(4):281-9.

26. Mehmeti B, Kelmendi J, Iiljazi-Shahiqi D, Azizi B, Jakovljevic S, Haliti $F$, et al. Comparison of shear bond strength orthodontic brackets bonded to zirconia and lithium disilicate crowns. Acta Stomatol Croat. 2019 Mar;53(1):17-27.

27. Douara Y, Abdul Kader S, Kassem H, Mowafy M. Evaluation of the shear bond strength of ceramic orthodontic brackets to glazed monolithic zirconia using different bonding protocols. Egypt Orthod J. 2019 Dec;56:9-20. 
28. Lee JY, Ahn J, An SI, Park JW. Comparison of bond strengths of ceramic brackets bonded to zirconia surfaces using different zirconia primers and a universal adhesive. Restor Dent Endod. 2018 Jan 22;43(1):e7.

29. Ihsan H, Al-Dabagh DJ. Comparison of shear bond strength of sapphire bracket bonded to zirconium surface after using different surface conditioning methods: in vitro study. J Baghdad Coll Dent. 2017 Sep;29(3):85-1.

30. Reynolds IR. A review of direct orthodontic bonding. Br J Orthodont. 1975;2(3):171-8.

31. Cavalcanti AN, Pilecki P, Foxton RM, Watson TF, Oliveira MT, Gianinni $M$, et al. Evaluation of the surface roughness and morphologic features of Y-TZP ceramics after different surface treatments. Photomed Laser Surg. 2009 Jun;27(3):473-9.

32. Varel H, Ashkenasi D, Rosenfeld A, Wähmer M, Campbell E. Micromachining of quartz with ultrashort laser pulses. Appl Phys A. 1997 Oct;65:367-73.

33. Gale MS, Darvell BW. Thermal cycling procedures for laboratory testing of dental restorations. J Dent. 1999 Feb;27(2):89-99. 